ORIGINAL ARTICLE

\title{
Suppression of human alloreactive T cells by linear tetrapyrroles; relevance for transplantation
}

\author{
SHAREE A. BASDEO, NICOLE K. CAMPBELL, LOUISE M. SULLIVAN, BRIAN FLOOD, \\ EMMA M. CREAGH, TIMOTHY J. MANTLE, JEAN M. FLETCHER ${ }^{1}$, and AISLING DUNNE ${ }^{1}$
}

DUBLIN 2, IRELAND

\begin{abstract}
The main limitation to successful transplantation is the antigraft response developed by the recipient immune system, and the adverse side effects of immunosuppressive agents which are associated with significant toxicity and counter indications such as infection and cancer. Furthermore, immunosuppressants do little to prevent ischemia-reperfusion injury during the transplantation procedure itself hence there is a growing need to develop novel immunosuppressive drugs specifically aimed at prolonging graft survival. Linear tetrapyrroles derived from the breakdown of mammalian heme have been shown in numerous studies to play a protective role in allograft transplantation and ischemia-reperfusion injury; however, commercial sources of these products have not been approved for use in humans. Plants and algae produce equivalent linear tetrapyrroles called bilins that serve as chromophores in light-sensing. One such marine-derived tetrapyrrole, phycocyanobilin (PCB), shows significant structural similarity to mammalian biliverdin (BV) and may prove to be a safer alternative for use in the clinic if it can exert direct effects on human immune cells. Using a mixed lymphocyte reaction, we quantified the allogeneic responses of recipient cells to donor cells and found that PCB, like BV, effectively suppressed proliferation and proinflammatory cytokine production. In addition, we found that $\mathrm{BV}$ and $\mathrm{PCB}$ can directly downregulate the proinflammatory responses of both innate dendritic cells and adaptive $T$ cells. We therefore propose that PCB may be an effective therapeutic drug in the clinical setting of transplantation and may also have wider applications in regulating inappropriate inflammation. (Translational Research 2016; [:1-14)
\end{abstract}

Abbreviations: $\mathrm{HO}-1$ = heme oxygenase; $\mathrm{BV}=$ biliverdin; $\mathrm{PCB}=$ phycocyanobilin

\section{INTRODUCTION}

mmunosuppressive therapy, although necessary after transplantation, is associated with many adverse consequences, including increased susceptibility to

\footnotetext{
${ }^{1}$ These authors contributed equally to this work.

From the School of Biochemistry and Immunology, Trinity Biomedical Sciences Institute, Trinity College Dublin, The University of Dublin, Dublin 2, Ireland; School of Medicine, Trinity Biomedical Sciences Institute, Trinity College Dublin, The University of Dublin, Dublin 2, Ireland.

Submitted for publication February 24, 2016; revision submitted June 16, 2016; accepted for publication July 13, 2016.
}

infection and cancer. Furthermore, immunosuppressants do little to prevent ischemia-reperfusion injury (IRI) or chronic rejection, and there has been a gap in the market over the last decade in the generation of

Reprint requests: School of Biochemistry and Immunology, Trinity Biomedical Sciences Institute, Trinity College Dublin, The University of Dublin, Room 3.10, Dublin 2, Ireland; e-mail: aidunne@tcd.ie.

1931-5244/\$ - see front matter

(C) 2016 Elsevier Inc. All rights reserved.

http://dx.doi.org/10.1016/j.trs1.2016.07.011 


\section{AT A GLANCE COMMENTARY}

Basdeo SA, et al.

Background

Patients receiving allografts require life-long immunosuppression which is often associated with increased risk of infection and cancer, hence, there is a need to develop less toxic treatment regimens specifically aimed at prolonging graft survival. Linear tetrapyrroles derived from the breakdown of mammalian heme play a protective role in allograft transplantation/ischemia-reperfusion injury however commercial sources of these products are not fit for human use.

\section{Translational Significance}

We demonstrate that a plant/algae derived tetrapyrrole, phycocyanobilin, significantly attenuates harmful pro-inflammatory responses to allogeneic cells and propose that it may be an effective therapeutic in the clinical setting of transplantation and dysregulated inflammation.

new therapies with safer side-effect profiles aimed at prolonging graft survival and combating oxidative damage. Several groups have reported that induction of the stress response protein, heme oxygenase-1 (HO-1), improves outcome after experimental transplantation and have attributed this protective effect to the heme breakdown products, carbon monoxide $(\mathrm{CO})$, and the tetrapyrroles, biliverdin (BV) and bilirubin. ${ }^{1-6}$ Administration of $\mathrm{BV}$ in a mouse model of heart transplantation significantly prolonged graft survival, resulted in enhanced tolerance to donor antigens and was accompanied by diminished immune cell infiltration in the grafts themselves. ${ }^{7}$ Furthermore, mesobiliverdin, a synthetic analogue of the seaweedderived linear tetrapyrrole, phycocyanobilin (PCB), was shown to enhance pancreatic islet yield and function in a model of islet transplantation which is currently being explored as a treatment option for type 1 diabetes. ${ }^{2}$ In addition to promoting tolerance, induction of HO-1 expression and administration of BV or bilirubin have also been reported to protect in models of IRI. ${ }^{1-6}$ For example, in a model of liver transplantation, after 16 hours of cold ischemia, rinse of the liver graft with bilirubin before reperfusion improved survival rate from $67 \%$ to $100 \%{ }^{8}$ Furthermore, adding $\mathrm{BV}$ to the preservation and perfusate solution itself, significantly improved graft function following transplantation. ${ }^{4}$ The protective effects of
$\mathrm{BV}$ and bilirubin are attributed to their ability to downregulate the production of proinflammatory cytokines from innate immune cells which in turn modulate adaptive $\mathrm{T}$ cell responses - the target of most immunosuppressive agents. In addition, BV and bilirubin are powerful antioxidants which most likely accounts for their protective effects during reperfusion when reactive oxygen species are known to be elevated.

Given the established immunomodulatory effects of linear tetrapyrroles in various animal models of disease, studies examining their effects on human immune cells are surprisingly few. Furthermore, little is known regarding the disease-ameliorating effects of plant/ algae-derived linear tetrapyrroles such as PCB. A recent study has, however, demonstrated that phycocyanin, which contains the active PCB moiety, has potent radical scavenging activity in human leukocytes and effectively reduces glucose oxidase-induced inflammation in the mouse paw suggesting that immunomodulatory activity is present. ${ }^{9}$ Commercially available BV, whether synthetic or extracted from bovine bile, has not been approved for human use and large scale production of BV in E.coli or yeast expression systems has been attempted but is hindered by potential endotoxin contamination. Therefore, plant/algae-derived tetrapyrroles such as PCB may prove to be a safer alternative for use in the clinic assuming they can exert direct effects on human immune cells.

Using an in vitro model of human allograft responses, we assessed the ability of BV and PCB to downregulate the harmful proinflammatory alloresponse of recipient peripheral blood mononuclear cells (PBMCs) to donor PBMC. In addition, we sought to determine the immune cell types through which these linear tetrapyrroles exert their effects. We found that BV and PCB significantly attenuated the harmful proinflammatory response to allogeneic cells and furthermore show that BV and PCB can exert direct effects on innate antigen presenting dendritic cells (DCs) and adaptive T cells, which both play a prominent role in chronic rejection post organ transplantation.

\section{MATERIALS AND METHODS}

Human blood samples. This study was approved by the research ethics committee of the School of Biochemistry and Immunology, Trinity College Dublin and is in accordance with the Declaration of Helsinki. Leukocyte-enriched buffy coats from anonymous healthy donors were obtained with permission from the Irish Blood Transfusion Service, St. James's Hospital, Dublin. PBMC were isolated by density gradient centrifugation (Lymphoprep; Axis-Shield poC). Cells were cultured in RPMI medium (Biosera) 
supplemented with 10\% FCS, 2-mM L-glutamine, 100$\mathrm{U} / \mathrm{ml}$ penicillin, and $100-\mu \mathrm{g} / \mathrm{ml}$ streptomycin (all Sigma Aldrich) and maintained in humidified incubators at $37^{\circ} \mathrm{C}$ with $5 \% \mathrm{CO}_{2}$.

Linear tetrapyrrole preparation for in vitro use. BV hydrochloride and PCB (both Inochem) were dissolved in 50-mM Tris $\mathrm{HCl}$ (Sigma Aldrich), $\mathrm{pH} 8.6$ and adjusted to $\mathrm{pH} 7.4$ for use in cell culture. Concentrations were determined by spectrophometry using the Beer Lambert law. The molar extinction coefficients for BV and PCB were $12500 \mathrm{M}^{-1} \mathrm{~cm}^{-1}$ and $13087 \mathrm{M}^{-1} \mathrm{~cm}^{-1}$, respectively. Stock solutions were prepared and then diluted as required. Vehicle controls (cells treated with Tris $\mathrm{HCl}, \mathrm{pH}$ 7.4) were assayed in conjunction with linear tetrapyrroles.

Cell viability and in vivo toxicity study. Cells were stained extracellularly with amine-binding markers for dead cells (viability dye; eBioscience). Cells were acquired on a BD FACS Canto II or LSR Fortessa (BD Biosciences) and analyzed with FlowJo software. Notably, BV, PCB, or the vehicle control showed no effect on cell viability at any concentration or time points tested. For the in vivo study, experiments were performed with 8- to 12-week-old female C57B1/6 mice bred under specific pathogen-free conditions, under license and approval of the local animal research ethics committee. To study the toxicity of PCB, C57B1/6 mice were treated with either PCB $(30 \mathrm{mg} / \mathrm{kg})$ or vehicle on days 0,3 , and 5 by oral gavage. The animals were monitored daily for weight loss. On the final experimental day, mice were humanely sacrificed and colons were harvested. Colon length was measured to confirm that colonic inflammation was not being induced by PCB.

In vitro stimulation with anti-CD3. Human PBMC, seeded at $2.5 \times 10^{5}$ per well in 96 well plates, were pretreated with $\mathrm{BV}, \mathrm{PCB}$, or vehicle control (Tris $\mathrm{HCl}$ ) at indicated concentrations for 3-6 hours before stimulation with anti-CD3 $(1 \mu \mathrm{g} / \mathrm{ml})$. Where indicated, cells were treated with $6.25-\mu \mathrm{M}$ tin protoporphyrin (SnPP; an inhibitor of HO-1). Unstimulated controls were assayed in parallel. Cell supernatants were analyzed by ELISA for the concentration of IFN- $\gamma$, IL-17, and TNF- $\alpha$ (all eBioscience Ready-Set-Go ELISA kits). Tritiated thymidine $(0.5 \mu \mathrm{Ci} /$ well $)$ was added 18 hours before the termination of the experiment, and proliferation was measured by incorporation of the radiolabel on a Wallac beta counter.

Assessment of antioxidant activity and BVR-A substrate specificity. The direct antioxidant capacities of the BV and PCB were assessed using trolox equivalent units as a standard. Briefly, working reagent was prepared according to the manufacturer's specification (Total Antioxidant Colorimetric Assay, Abcam) and added to either a trolox standard or the samples to be determined. The samples were assayed at $100 \mu \mathrm{M}$, as determined by spectrophotometry. A standard curve of trolox concentration as a function of absorbance at $570 \mathrm{~nm}$ was generated and used to calculate the antioxidant capacity of the samples. In addition, the antioxidant capacity of known antioxidant compounds, glutathione and $\alpha$-tocopherol, was also determined. BV-IX $\alpha$ reductase (BVR) activity was measured by monitoring the production of bilirubin or phycocyanorubin at $460 \mathrm{~nm}$ and $420 \mathrm{~nm}$ respectively. Assays were conducted at room temperature in the presence of $1 \mathrm{mg} / \mathrm{mL}$ BSA, $50-\mu \mathrm{M}$ NADPH, and increasing concentrations of BV and PCB (from 0 to $20 \mu \mathrm{M}$ ). BVR was added at a concentration of $300 \mathrm{ng} / \mathrm{mL}$. The assay was conducted in 100-mM Tris, $\mathrm{pH} 8.5$.

Human in vitro primary and secondary alloresponse assays. The immunomodulatory potential of linear tetrapyrroles on human primary and secondary alloresponses was tested in vitro using a unidirectional mixed lymphocyte reaction. To assess primary alloresponses, $2.5 \times 10^{5}$ human PBMC or $1 \times 10^{5} \mathrm{CD} 4$ $\mathrm{T}$ cells were pretreated with $\mathrm{BV}(50 \mu \mathrm{M}), \mathrm{PCB}$ $(50 \mu \mathrm{M})$, or vehicle control for 3-6 hours, stimulated with $2.5 \times 10^{5}$ irradiated (60 Gy) allogeneic PBMC or irradiated autologous PBMC as a negative control. Cells were maintained in humidified incubators at $37^{\circ} \mathrm{C}$ with $5 \% \mathrm{CO}_{2}$ for 7 days. For secondary alloresponses, human PBMC or CD4 T cells were stimulated with irradiated allogeneic PBMC for 7 days (primary alloresponse) and were allowed to rest for a further 5 days before washing off BV, PCB, or vehicle control. Cells were then restimulated with the same irradiated allogeneic PBMC as those used to stimulate the primary alloresponse or with anti-CD3 and anti-CD28, as a control. No linear tetrapyrroles were added to the secondary alloresponse. Cells were maintained in humidified incubators at $37^{\circ} \mathrm{C}$ with $5 \% \mathrm{CO}_{2}$ for a further 5-6 days. Cell supernatants were harvested for ELISA to determine the concentrations of IFN- $\gamma$, IL-17, TNF- $\alpha$, and GM-CSF (all eBioscience Ready-Set-Go ELISA kits $)$. Tritiated thymidine $(0.5 \mu \mathrm{Ci} /$ well $)$ was added 18 hours before the termination of the experiment to measure allospecific proliferation. Proliferation was measured by incorporation of the radiolabel on a Wallac beta counter. Only responses greater than twice the autologous response were evaluated.

CD4 T cell assays. CD4 T cells were positively selected from PBMC by using anti-CD4-microbeads (Miltenyi Biotech; according to the manufacturer's protocol). Cells were pretreated with BV, PCB, or vehicle control (Tris $\mathrm{HCl}$ ) at indicated concentrations for 3-6 hours before stimulation with anti-CD3 $(1 \mu \mathrm{g} / \mathrm{ml})$ and anti-CD28 $(0.5 \mu \mathrm{g} / \mathrm{ml})$ for 4 days. Unstimulated 
controls were assayed in parallel. Cell supernatants were analyzed by ELISA for the concentration of IFN- $\gamma$ and IL-17 (eBioscience Ready-Set-Go ELISA kits). Tritiated thymidine $(0.5 \mu \mathrm{Ci} /$ well $)$ was added 18 hours before the termination of the experiment and proliferation was measured by incorporation of the radiolabel on a Wallac beta counter.

Treg induction and suppressive capacity. PBMC were treated with $\mathrm{PCB}$ or vehicle control before stimulation with irradiated allogeneic PBMC to elicit a primary alloresponse as aforementioned. On day 12, cells were washed and vehicle control-treated cells were stained with CFSE. CFSE-labeled vehicle control cells were cultured alone or in coculture with equal numbers of cells that had been treated with PCB. Cells were restimulated with the same irradiated allogeneic donor used to initiate the primary alloresponse and were maintained in humidified incubators at $37^{\circ} \mathrm{C}$ with $5 \%$ $\mathrm{CO}_{2}$ for 5 days. Cells were stained with fluorochromeconjugated antibodies specific for CD4, CD25, CD127, and FoxP3 and analyzed by flow cytometry. The frequencies of Treg cells $\left(\mathrm{CD} 4^{+} \mathrm{CD} 127^{\mathrm{lo}} \mathrm{CD} 25^{+}\right.$ $\mathrm{FoxP}^{+}$) in CFSE-labeled cells (cells treated with vehicle control only during the primary alloresponse) and in unlabeled cells (cells treated with PCB during the primary alloresponse) were assessed. Proliferation in response to secondary allogeneic restimulation of cells treated with vehicle control during the primary response was assessed by CFSE dilution and by incorporation of tritiated thymidine. Cell supernatants were harvested for ELISA to determine the concentration of IFN- $\gamma$ present (eBioscience ReadySet-Go ELISA kit).

Dendritic cell assays. $\mathrm{CD} 14^{+}$monocytes were positively selected from PBMC by using anti-CD14microbeads (Miltenyi Biotech; according to the manufacturer's protocol). Monocytes were cultured at $1 \times 10^{6}$ cells $/ \mathrm{ml}$ with IL-4 (40 ng/ml) and GM-CSF (50 ng/ml; both Miltenyi Biotech) for 7 days. Cells were fed on day 3 with medium supplemented with cytokines. Non-adherent DC were harvested by centrifugation and cultured at $1 \times 10^{6}$ cells $/ \mathrm{ml}$. The purity of $\mathrm{CD} 14^{\mathrm{lo}} \mathrm{DC}-\mathrm{SIGN}^{+} \mathrm{DC}$ was assessed by flow cytometry and was routinely $>98 \%$. DC were cultured at $1 \times 10^{6}$ cells $/ \mathrm{ml}$ in the presence of $50-\mu \mathrm{M}$ $\mathrm{BV}, \mathrm{PCB}$, or vehicle control and stimulated with ultrapure LPS (100 ng/ml; Invivogen). After 24 hours, cells were stained with fluorochrome-conjugated antibodies specific for CD83, CD40, CD80, and HLADR. Cells were acquired on a BD FACS Canto II or LSR Fortessa (BD Biosciences) and analyzed with FlowJo software. Supernatants were analyzed by ELISA to quantify the concentration of IL-12p70 and IL-23 (both eBioscience Ready-Set-Go ELISA kits).
For DC-T cell coculture experiments, CD4 T cells were positively selected from PBMC using anti-CD4 microbeads (Miltenyi Biotech; according to the manufacturer's protocol) with purities routinely $>95 \%$. CD4 T cells were cocultured with allogeneic DC that had been pretreated with BV, PCB, or vehicle control then activated with LPS $(100 \mathrm{ng} / \mathrm{ml}$; Invivogen) for 24 hours and thoroughly washed. Cells were cocultured at a ratio of $10 \mathrm{CD} 4 \mathrm{~T}$ cells to $1 \mathrm{DC}$ with no linear tetrapyrroles present for 5 days. Supernatants were analyzed by ELISA to quantify the concentration of IFN- $\gamma$ (eBioscience Ready-Set-Go ELISA kits).

Statistical analysis. Statistical analyses were performed using Prism 5 software; comparison of 2 treatment groups within a sample were determined by Student paired $t$ test with 2 -tailed $P$-values, $\geq 3$ groups were analyzed by 1-way ANOVA with Tukey's multiple comparison post-test. Statistical differences between 2 groups, each containing more than one variable, were determined by 2-way ANOVA with Bonferroni post-tests; $P$ values $\leq 0.05$ were considered significant and denoted with asterisks in the figures.

\section{RESULTS}

Marine-derived phycocyanobilin and mammalian biliverdin significantly reduce the proliferation and cytokine production of human PBMC in response to antiCD3. While it is established that mammalian BV is potently anti-inflammatory in animal models, its effects have been understudied in human cells. Moreover, marine-derived PCB, has come into prominence for its potential as a therapeutic alternative to mammalian linear tetrapyrroles; however, its effect on human cells and its efficacy compared with BV has not yet been studied. We sought to determine if $\mathrm{PCB}$ and $\mathrm{BV}$ have similar anti-inflammatory effects on human cells in vitro. Human $\mathrm{PBMC}$ were pretreated with $\mathrm{BV}$ or PCB and stimulated with anti-CD3. Proliferation was measured by incorporation of radiolabelled thymidine into DNA during mitosis. In cells pretreated with BV or $\mathrm{PCB}$, the proliferation in response to anti-CD3 was significantly attenuated in a dose-dependent manner (Fig 1, $A$ and $B$ ). Notably, BV and PCB had no effect on the viability of the lymphocytes (Fig $1, A$ and $B$, right panel; Supplemental Fig $1, A$ and $B$ ). Furthermore, we assessed the tolerability of PCB in vivo given that there are no studies assessing potential adverse effects following the consumption of this product which is a recognized component of seaweed-based supplements. $\mathrm{C} 57 \mathrm{Bl} / 6$ mice were treated with either PCB $(30 \mathrm{mg} / \mathrm{kg})$ or vehicle control by oral gavage 3 times over the course of 7 days (day 
A

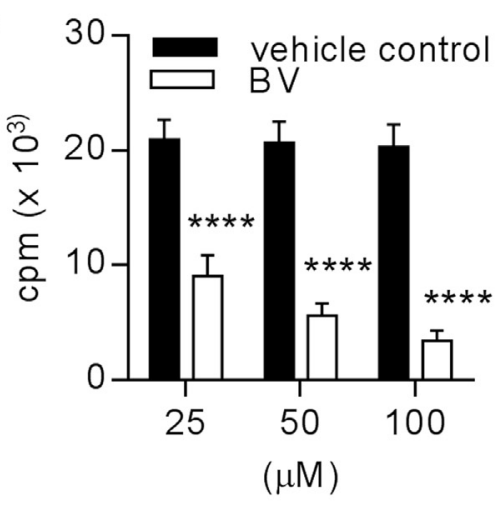

B

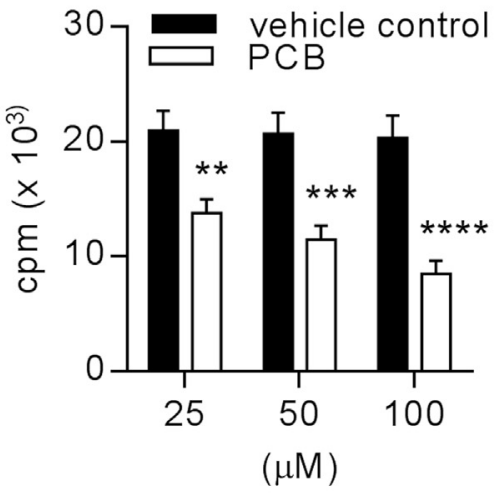

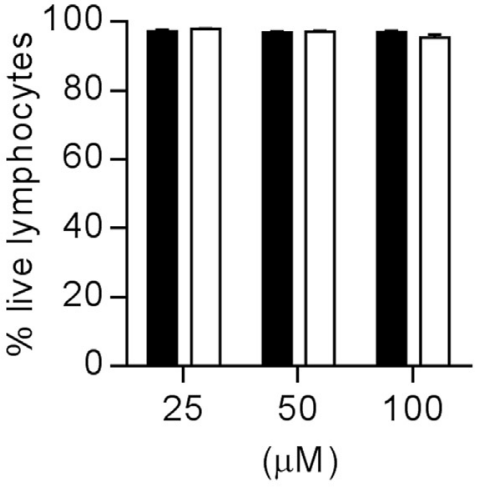

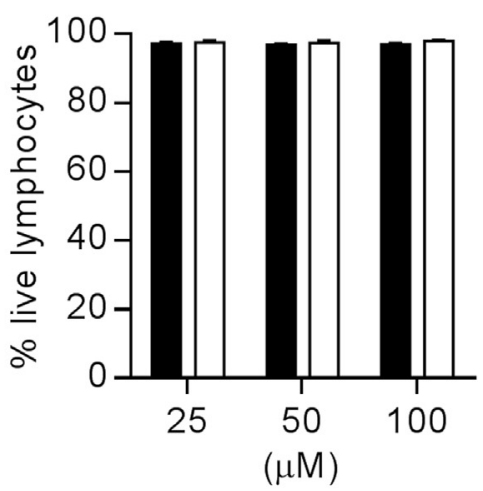

C
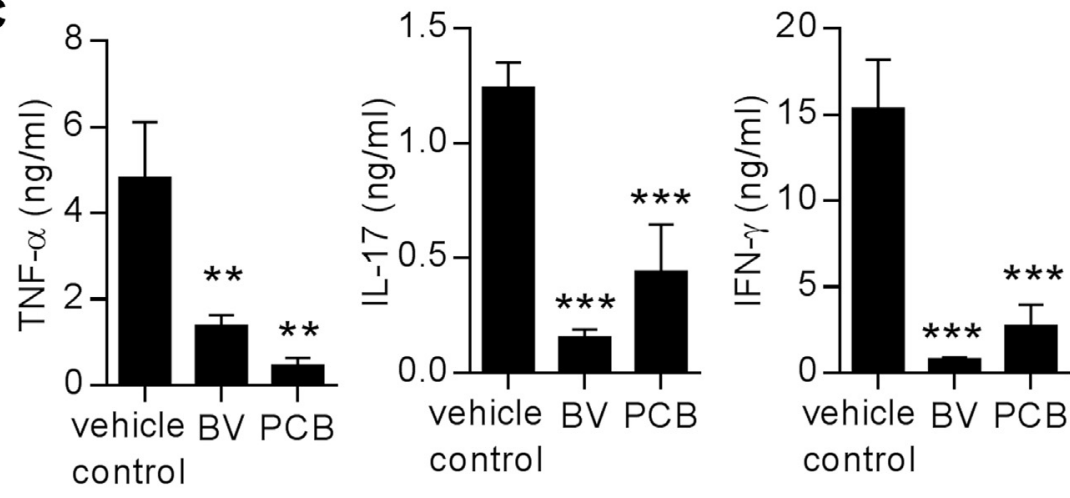

D

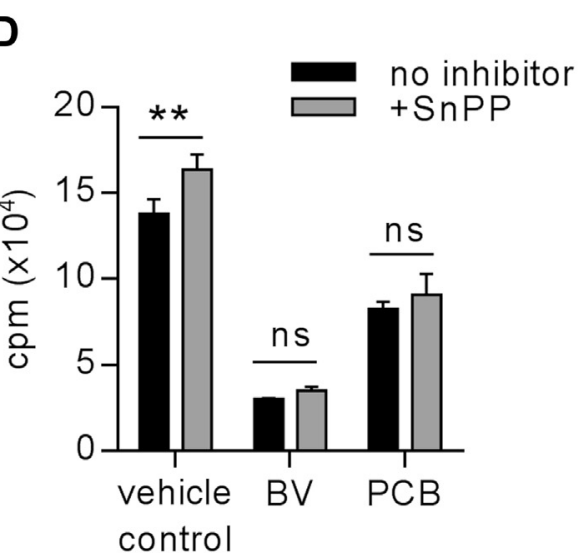

E

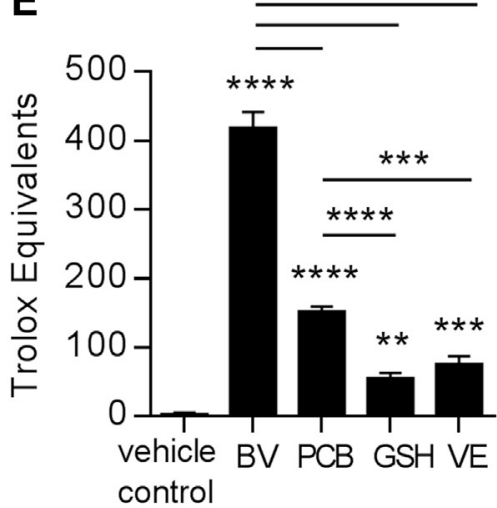

Fig 1. BV and PCB significantly reduce proliferation and cytokine production from PBMC, independently of HO-1 induction, and exhibit potent antioxidant capacity. PBMC were isolated from healthy donors $(\mathrm{n}=7)$ and treated with biliverdin $(\mathrm{BV})$, phycocyanobilin $(\mathrm{PCB})$, or vehicle control (Tris $\mathrm{HCl}$ at $\mathrm{pH} 7.4$ ) for $6 \mathrm{~h}$ before 
0,3 , and 5). The animals were monitored daily for weight loss and diarrhea. No significant differences were observed between mice that were treated with PCB compared with controls (Supplemental Fig 1, C). On the final experimental day, mice were humanely sacrificed and colons were harvested. PCB-treated mice had similar colon lengths compared with vehicle control-treated mice and colonic inflammation was not evident (Supplemental Fig 1, D).

The production of proinflammatory cytokines; TNF- $\alpha$, IFN- $\gamma$, and IL-17 from PBMC pretreated with $100-\mu \mathrm{M}$ BV or PCB and stimulated with anti-CD3 were measured by ELISA (Fig 1,C). Both BV and PCB significantly reduced the production of all proinflammatory cytokines analyzed. No significant differences were observed between the concentrations of cytokines present in BV-treated cells compared with PCB-treated cells (Fig 1,C). Furthermore, we found that the compounds did not affect the ability of $\mathrm{T}$ cells to produce IL-2, suggesting that the compounds are not having a global inhibitory effect on cytokine production (Supplemental Fig 2). These data indicate that marine-derived PCB and mammalian BV are highly immunosuppressive as they significantly attenuated proliferation (albeit, independently of IL-2) and inflammatory cytokine production from human PBMC in response to polyclonal T-cell receptor (TCR) activation in vitro.

To determine if $\mathrm{BV}$ and $\mathrm{PCB}$ are exerting their effects via HO-1, cells were pretreated with the tetrapyrroles or vehicle control in the presence or absence of tin protoporphyrin (SnPP), a known inhibitor of HO-1. Cells were then stimulated with anti-CD3 for 3 days as aforementioned and proliferation was assessed by incorporation of tritiated thymidine. In cells treated with vehicle alone, SnPP significantly increased the proliferation of PBMC in response to anti-CD3. However, in cells treated with BV or PCB, SnPP had no effect (Fig 1, $D)$. In addition, neither BV nor PCB were capable of inducing HO-1 expression compared with the positive control, cobalt protoporphyrin IX (data not shown).
This indicates that the mechanism by which BV and PCB modulate inflammation is independent of the induction of HO-1.

Biliverdin and phycocyanobilin exhibit strong antioxidant capacity which may protect against ischemia-reperfusion injury in transplant recipients. Both BV and PCB have established antioxidant ability; however, their antioxidant capacity has not yet been directly compared. The antioxidant capacities of BV and PCB were determined using a Trolox-based colorimetric assay. BV and PCB were compared with vehicle control and known antioxidants; glutathione (GSH) and $\alpha$-tocopherol (the active form of vitamin E; VE). Results demonstrate that all compounds tested have significantly increased antioxidant activity compared with the vehicle control (Fig 1,E). Interestingly, both $\mathrm{BV}$ and $\mathrm{PCB}$ have significantly greater antioxidant capacity than GSH and VE. However, BV demonstrated significantly more antioxidant activity compared with PCB. These data demonstrate that $\mathrm{BV}$ and $\mathrm{PCB}$ have potent antioxidant activity and, together with their antiinflammatory properties shown above, indicate that these linear tetrapyrroles may be effective as immunosuppressant drugs to induce tolerance to transplanted organs and limit IRI.

In mammalian cells, BV is converted to bilirubin (also considered a powerful antioxidant) via the action of $\mathrm{BV}-\mathrm{IX} \alpha$ reductase (BVR-A). It has previously been demonstrated that PCB is a substrate for rat liver BVR-A and is rapidly converted to phycocyanorubin. ${ }^{10}$ We next carried out an enzyme assay in order to determine if $\mathrm{PCB}$ is a substrate for the human form of BVR-A. We found that PCB is indeed a substrate for the human enzyme, howev$\mathrm{er}$, it is reduced at a lower rate $(\mathrm{Vmax}=1.28 \mu \mathrm{mol} /$ $\mathrm{min} / \mathrm{mg}$ for PCB vs $8.3 \mu \mathrm{mol} / \mathrm{min} / \mathrm{mg}$ for BV) and the enzyme has an approximately 5-fold lower affinity for PCB compared with the natural BV substrate $(\mathrm{Km}=1.25 \mu \mathrm{M}$ vs $0.25 \mu \mathrm{M}$ for $\mathrm{PCB}$ and $\mathrm{BV}$, respectively). This data does however, suggest

stimulation with anti-CD3 for $72 \mathrm{~h}$. Proliferation was determined by incorporation of tritiated thymidine (measured in counts per minute; $\mathrm{cpm}$ ) which was added to wells for the final $18 \mathrm{~h}$ of stimulation. Cells were stained with a viability dye and analyzed by flow cytometry. (A) The proliferation (left) and viability (right) of cells treated with biliverdin compared with vehicle control. (B) The proliferation (left) and viability (right) of cells treated with PCB compared with vehicle control. (C) The concentrations of cytokines present in the supernatants of cells treated with $100 \mu \mathrm{M}$ of biliverdin or PCB compared with vehicle control were analyzed by ELISA. (D) PBMCs were treated with 50- $\mu \mathrm{M}$ BV or PCB in the presence or absence of SnPP, stimulated with anti-CD3, and proliferation was determined by incorporation of tritiated thymidine, as above. (E) Antioxidant capacity was determined using a Trolox-based colorimetric assay. Biliverdin and PCB were compared with vehicle control and known antioxidants; glutathione (GSH) and $\alpha$-tocopherol (the active form of vitamin E; VE). Results are collated from 3 independent experiments. Statistical significance was determined by 2-way ANOVA with Bonferroni's post-test $(\mathbf{A}, \mathbf{B}$, and $\mathbf{D})$ or 1-way ANOVA with Tukey's multiple comparisons post-test $(\mathbf{C}$ and $\mathbf{E}) ; * * * * P<.0001$, $* * * P<.001, * * P<.01$. PBMC, peripheral blood mononuclear cell. 

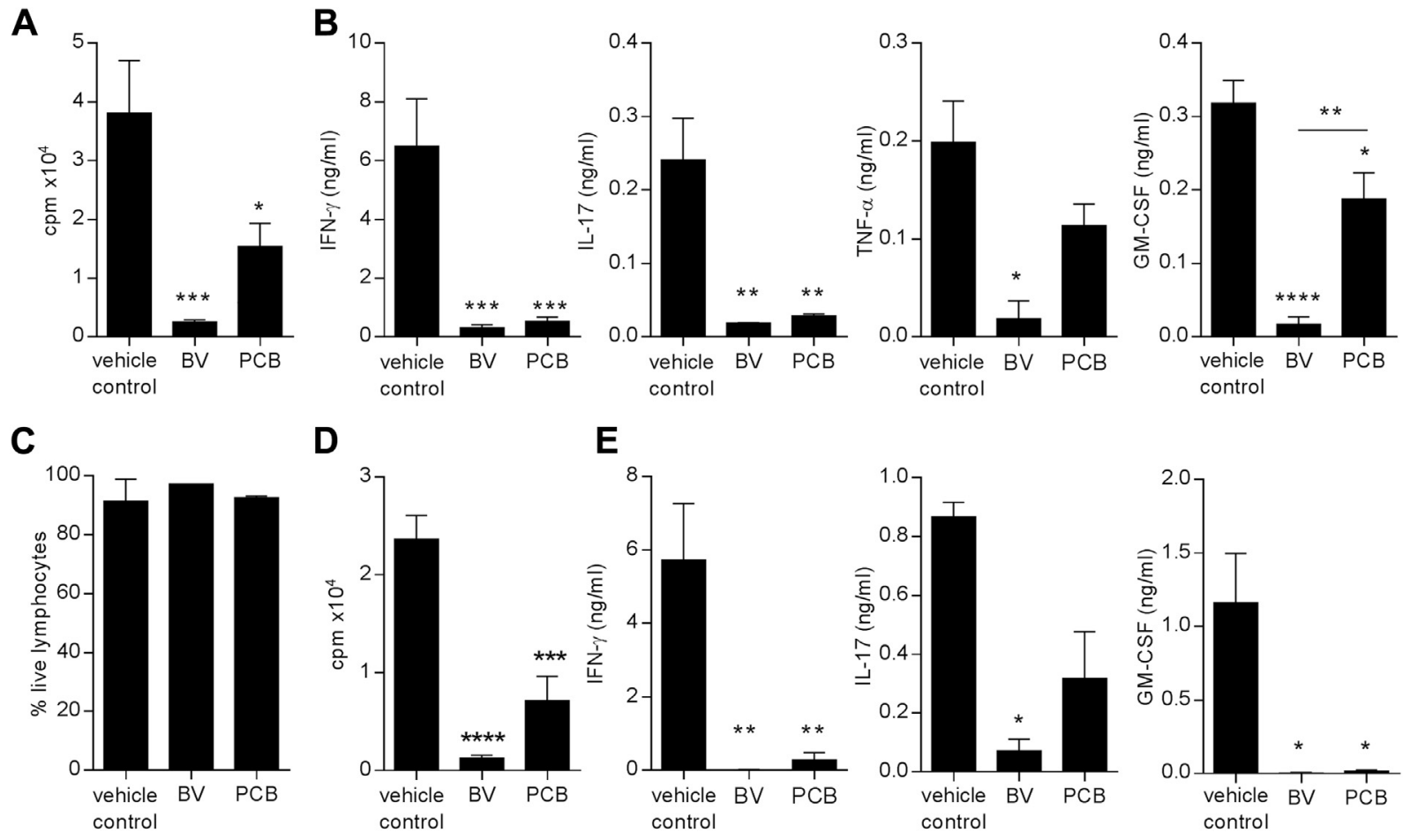

D

E
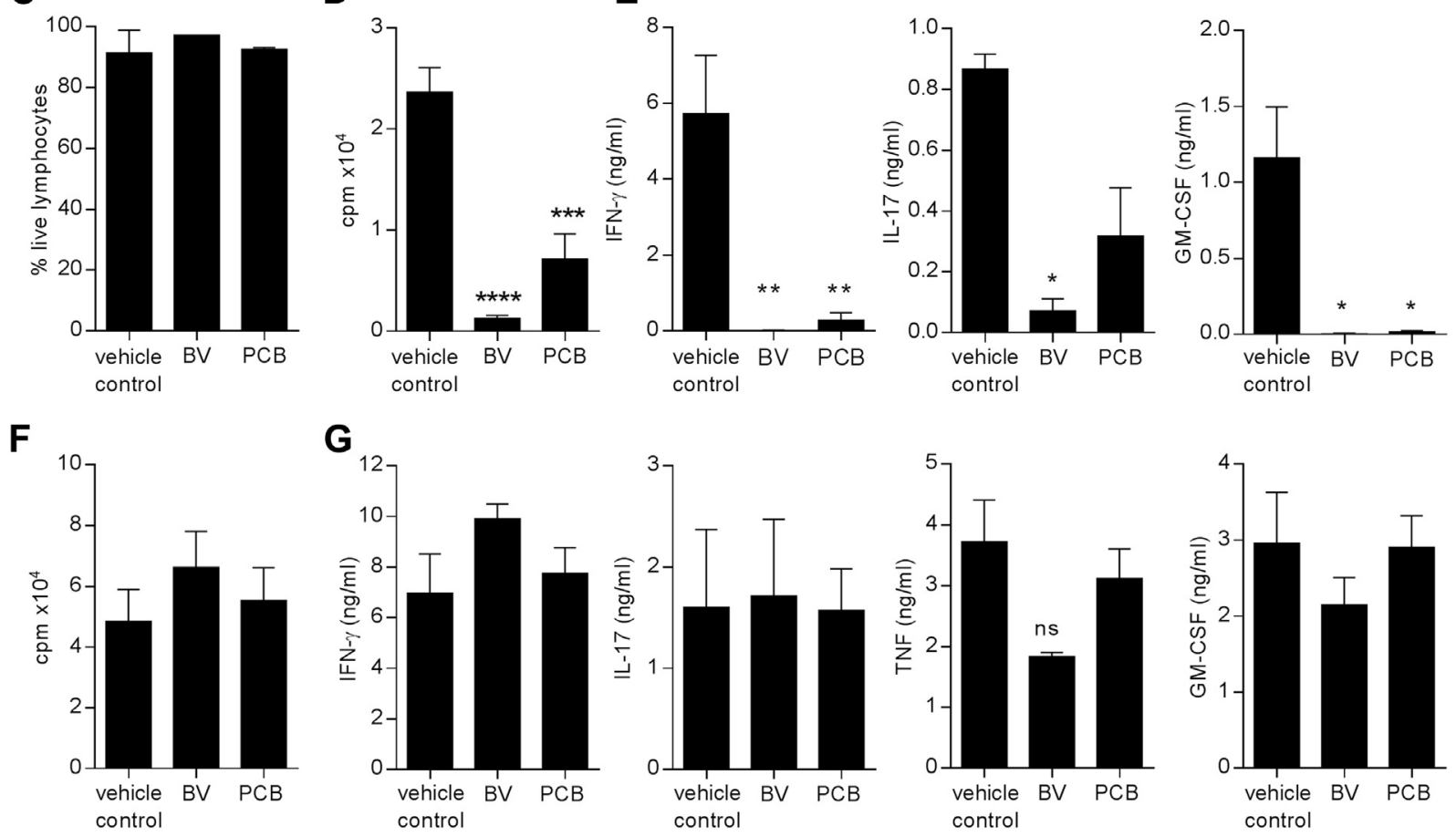

Fig 2. Biliverdin and phycocyanobilin attenuate primary and secondary allogeneic proinflammatory responses but not mitogen-specific responses in human PBMC. PBMC were isolated from healthy donors and treated with biliverdin (BV), phycocyanobilin (PCB), or vehicle control for $3 \mathrm{~h}$ before the addition of irradiated allogeneic PBMC for $7 \mathrm{~d}$. Proliferation was assessed by measuring the incorporation of tritiated thymidine. (A) The proliferative primary alloresponses (measured in counts per minute; cpm) of PBMC $(n=9)$. (B) The concentrations of proinflammatory cytokines; IFN- $\gamma$, IL-17, TNF- $\alpha$, and GM-CSF were measured by ELISA; ( $n=3-6)$. To assess secondary allorestimulation, $\mathrm{PBMC}$ treated with $\mathrm{BV}, \mathrm{PCB}$, or vehicle control were allowed to rest for $5 \mathrm{~d}$ post primary stimulation (ie, until day 12). (C) Cell viability was assessed by flow cytometry on day 12. (D) Cells were washed and restimulated with the same irradiated allogeneic PBMC or stimulated with anti-CD3 and anti-CD28 for $5 \mathrm{~d}$. Tritiated thymidine was added for the final $18 \mathrm{~h}$ to measure proliferation. The proliferation of PBMC which had undergone primary allogeneic stimulation and were subsequently restimulated with the same irradiated allogeneic PBMC (D; n = 4). (E) Representative graphs show the concentrations of IFN- $\gamma$, IL-17, and GM-CSF measured by ELISA for PBMC which had undergone secondary allogeneic stimulation. (F) The proliferation of PBMC which had undergone primary allogeneic stimulation and were subsequently restimulated with antiCD3 and anti-CD28 $(\mathrm{n}=4)$. (G) The concentrations of proinflammatory cytokines; IFN- $\gamma$, IL-17, TNF- $\alpha$, and GM-CSF were measured by ELISA in PBMC which had been restimulated with anti-CD3 and anti-CD28 $(\mathrm{n}=4)$. Statistical significance was determined by 1-way ANOVA with Tukey's multiple comparisons posttest; $* * * P<.0001,{ }^{* * *} P<.001, * * P<.01, * P<.05$. PBMC, peripheral blood mononuclear cell.

that PCB is converted to rubin in human cells and can likely exert antioxidant activity via both molecules.
Biliverdin and phycocyanobilin exert potent immunosuppressive effects on primary and secondary alloresponses in PBMC. Having established that BV and 
PCB exert potent anti-inflammatory and antioxidant effects in human cells, we next sought to determine if BV and PCB could attenuate alloresponses which cause harmful inflammation in the context of transplantation. PBMC or CD4 $\mathrm{T}$ cells were isolated from healthy donors and pretreated with $\mathrm{BV}, \mathrm{PCB}$, or vehicle control before stimulation with allogeneic irradiated PBMC. The primary alloresponse to allogeneic donor cells was evaluated on day 7; proliferation was assessed by incorporation of tritiated thymidine and the concentrations of proinflammatory cytokines present in the supernatants were determined by ELISA. Both BV and PCB significantly attenuated proliferation in response to allogeneic PBMC, with $\mathrm{BV}$ exhibiting a stronger effect than PCB; however, this difference was not statistically significant (Fig 2, A). BV significantly suppressed the production of all proinflammatory cytokines tested, whereas PCB exhibited significant suppression of IFN- $\gamma$, IL-17, and GM-CSF and a trend toward reduced production of TNF- $\alpha$ (Fig 2, B). These data show that BV and PCB significantly attenuated primary alloresponses and may therefore be effective immunosuppressants to combat acute organ rejection. However, chronic rejection, the main cause of allograft failure in the clinic, occurs when $\mathrm{B}$ and $\mathrm{T}$ cells are activated and reactivated over protracted periods of time.

To ascertain if the anti-inflammatory effects of BV and $\mathrm{PCB}$ were sufficient to maintain a state of tolerance to a secondary allostimulation, cells exposed to a primary allostimulation in the presence of $\mathrm{BV}$ and PCB for 7 days were allowed to rest for 5 days. On day 12 post primary allostimulation, cell culture supernatants containing $\mathrm{BV}, \mathrm{PCB}$, or vehicle control were removed, and cells were washed and stimulated with fresh irradiated allogeneic PBMC from the same donor used in the primary alloresponse, in the absence of linear tetrapyrroles. The viability of lymphocytes (gated on the basis of forward and side scatter) was tested on day 12 before restimulation; all lymphocytes were approximately $90 \%$ viable across all treatment groups (Fig 2, C). After 6 days, the proliferation and cytokine production of cells exposed to secondary allorestimulation were analyzed; PBMC treated with $\mathrm{BV}$ and PCB during primary allostimulation exhibited significantly reduced proliferation and production of IFN- $\gamma$ and GM-CSF in response to secondary allorestimulation even in the absence of BV or PCB (Fig 2, $D$ and $E$ ). $\mathrm{BV}$ treatment during primary allostimulation significantly reduced the production of IL-17 from PBMC during secondary allorestimulation, whereas PBMC initially treated with PCB exhibited a trend toward reduced IL-17; however, this result was not statistically significant (Fig 2, E). These data indicate that treatment with $\mathrm{BV}$ or $\mathrm{PCB}$ significantly reduced harmful proinflammatory primary alloresponses in human PBMC and maintained the cells in a tolerogenic state during a secondary allostimulation in the absence of $\mathrm{BV}$ or $\mathrm{PCB}$.

To determine if the tolerance to secondary allogeneic responses induced by treatment with linear tetrapyrroles during the primary response was specific to the alloantigen or nonspecific global dampening of the immune response, cells that had undergone primary alloresponses were restimulated with a mitogenic stimulus; anti-CD3 and anti-CD28. Proliferation was assessed by incorporation of tritiated thymidine (Fig 2, F). Importantly, when these cells underwent secondary restimulation with a nonspecific mitogen, no differences were observed in the proliferation of cells that had been previously treated with vehicle control, BV, or PCB. Furthermore, the production of proinflammatory cytokines in response to the mitogenic secondary restimulation was not significantly different between the groups (Fig 2, G). These results suggest that while cells treated with BV and PCB during a primary allogeneic stimulation attenuate proinflammatory responses to secondary allogeneic stimulation, this is specific to the allogeneic antigens as cells retain the ability to respond to mitogen-specific stimulation.

Biliverdin and phycocyanobilin suppress responses to primary and secondary allostimulation in CD4 T cells. We next sought to determine if $\mathrm{BV}$ and PCB could have a direct effect on CD4 T cells, within the mixed lymphocyte reaction, that are instrumental in propagating chronic inflammation through the production of proinflammatory mediators and the provision of help to $\mathrm{B}$ cells. CD4 T cells were isolated from healthy donors and, similar to the previous experiment examining alloresponses in total PBMC, CD4 T cells were pretreated with $\mathrm{BV}, \mathrm{PCB}$, or vehicle control before stimulation with allogeneic-irradiated PBMC. The proliferation of $\mathrm{CD} 4 \mathrm{~T}$ cells in response to irradiated allogeneic PBMC was significantly attenuated in the presence of $\mathrm{BV}$ and $\mathrm{PCB}$, with BV exhibiting markedly increased capacity to reduce proliferation compared with $\mathrm{PCB}$ (Fig 3, A). Both BV and PCB significantly reduced the production of proinflammatory cytokines from allostimulated CD4 $\mathrm{T}$ cells with no significant difference observed between the extent of reduction induced by $\mathrm{BV}$ or $\mathrm{PCB}$ (Fig 3, B). On secondary restimulation in the absence of $\mathrm{BV}$ or $\mathrm{PCB}, \mathrm{CD} 4$ $\mathrm{T}$ cells treated with $\mathrm{BV}$ or $\mathrm{PCB}$ during primary allostimulation exhibited significantly reduced proliferation and production of IFN- $\gamma$ and GM-CSF (Fig 3, $C$ and D). Similarly, CD4 T cells treated with BV during the primary allostimulation showed a significant reduction in IL-17 production, whereas 
A

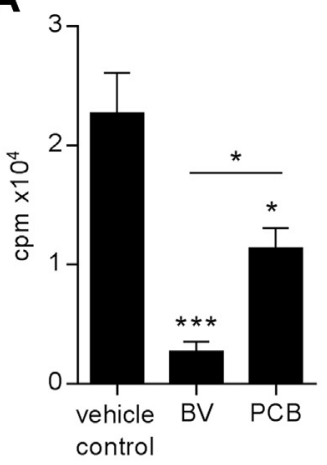

C

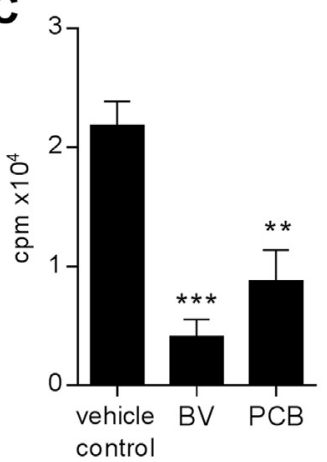

E

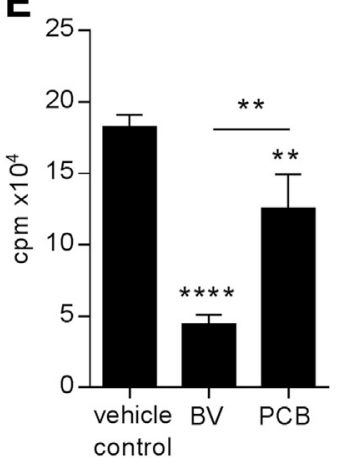

B
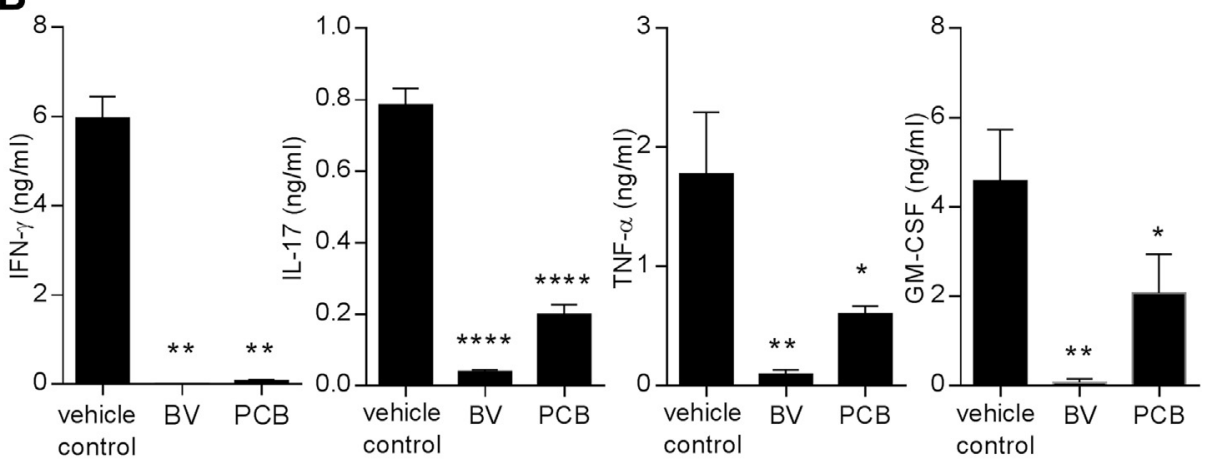

D
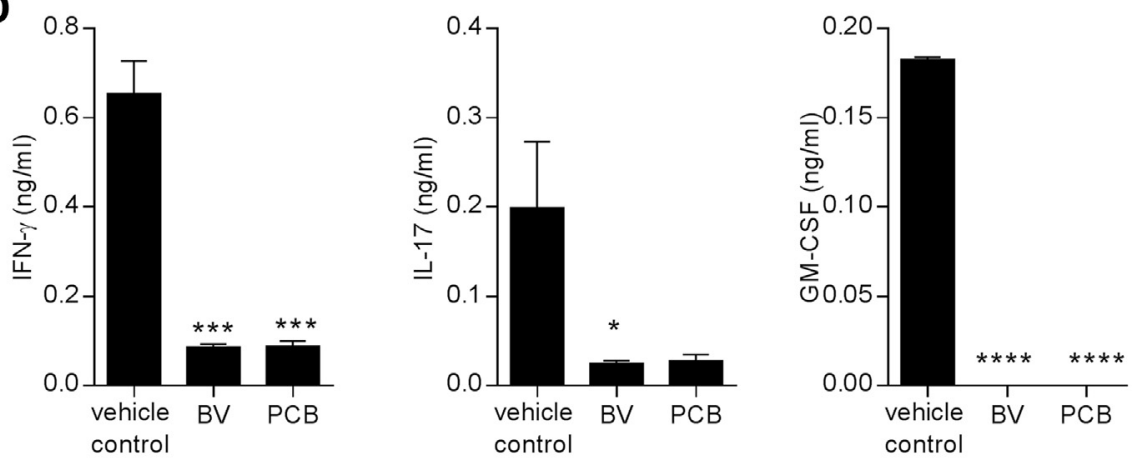

$\mathbf{F}$

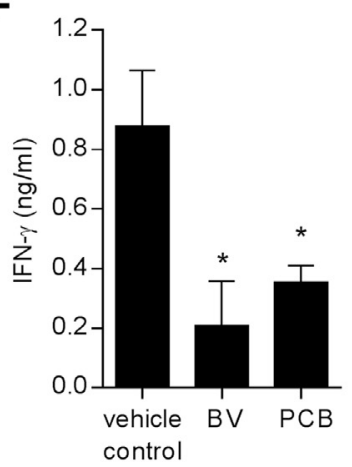

Fig 3. Biliverdin and phycocyanobilin attenuate primary and secondary allogeneic proinflammatory responses in human CD4 T cells. PBMC were isolated from healthy donors, and CD4 T cells were purified by magnetic sorting. Cells were treated with BV, PCB, or vehicle control for $6 \mathrm{~h}$ before the addition of irradiated allogeneic PBMC for $7 \mathrm{~d}$. (A) The proliferative primary alloresponses (measured in counts per minute; cpm) of CD4 ${ }^{+} \mathrm{T}$ cells $(\mathrm{n}=5$ ). (B) Representative ELISA data show the concentrations of IFN- $\gamma$, IL-17, TNF- $\alpha$, and GM-CSF present in the supernatants. To assess secondary allorestimulation, $\mathrm{CD} 4{ }^{+} \mathrm{T}$ cells treated with $\mathrm{BV}, \mathrm{PCB}$, or vehicle control were allowed to rest for $5 \mathrm{~d}$ post primary stimulation (ie, until day 12). Cells were washed and restimulated with the same irradiated allogeneic PBMC for $6 \mathrm{~d}$. Tritiated thymidine was added for the final $18 \mathrm{~h}$ to measure proliferation in $\mathrm{CD}^{+} \mathrm{T}$ cells $(\mathbf{C} ; \mathrm{n}=4)$. (D) Representative graphs show the concentrations of IFN- $\gamma$, IL-17, and GM-CSF measured by ELISA. CD4 T cells were purified by magnetic sorting and treated with BV, PCB, or vehicle control for $6 \mathrm{~h}$ before stimulation with anti-CD3 and anti-CD28. (E) Proliferation was determined by incorporation of tritiated thymidine which was added to wells for the final $18 \mathrm{~h}$ of stimulation. (F) The concentrations of IFN- $\gamma$ and IL-17 present in the supernatants were determined by ELISA. Statistical significance was determined by 1way ANOVA with Tukey's multiple comparisons post-test; **** $P<.0001,{ }^{* * *} P<.001,{ }^{*} P<<.01, * P<.05$. PBMC, peripheral blood mononuclear cell.

cells previously treated with PCB exhibited a trend toward reduced production of IL-17 in the secondary alloresponse (Fig 3,D). These data demonstrate that treatment with $\mathrm{BV}$ and $\mathrm{PCB}$ significantly reduced harmful proinflammatory primary alloresponses in human CD4 $\mathrm{T}$ cells and maintained the cells in a tolerogenic state during a secondary allostimulation in the absence of BV or PCB. Thus, these data indicate 
A

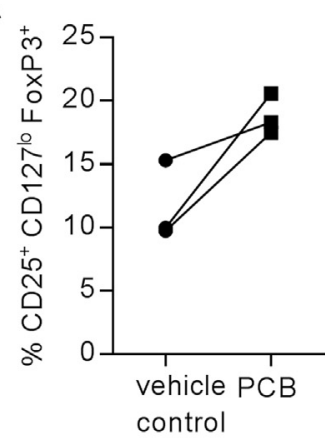

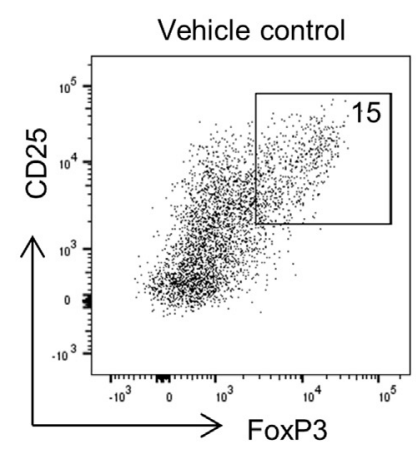

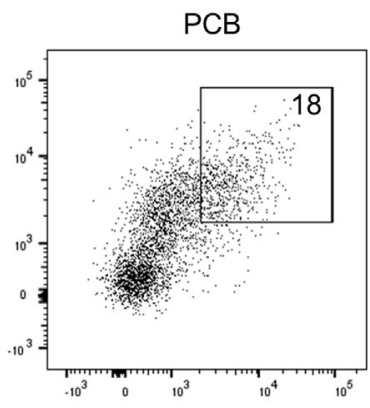

B

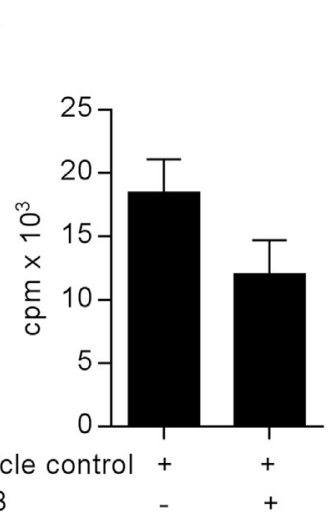

PCB

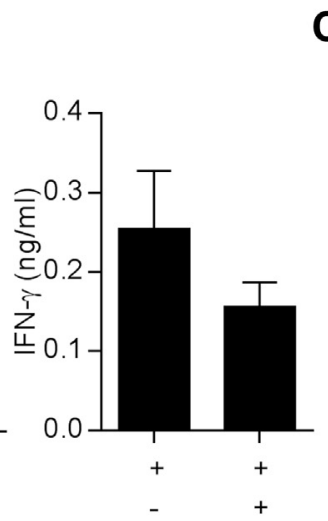
control cells
alone

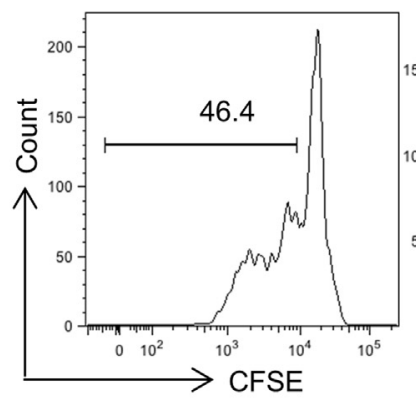

control cells co-cultured with PCB treated cells

Fig 4. Cells treated with phycocyanobilin during primary allogeneic stimulation exhibit a trend toward an increased frequency of Treg cells and suppressive capacity. PBMC were isolated from healthy donors and treated with PCB or vehicle control for $3 \mathrm{~h}$ before the addition of irradiated allogeneic PBMC for $12 \mathrm{~d}$. Cells were washed and vehicle control-treated cells were labeled with CFSE and cultured alone or cocultured with equal numbers of cells that had been treated with PCB. Cells were stimulated with the same irradiated allogeneic donor as used in the primary alloreponse for a further $5 \mathrm{~d}$. (A) Cells were stained with fluorochrome-conjugated antibodies specific for CD4, CD25, CD127, and FoxP3 and analyzed by flow cytometry. The graph shows the frequency of CD4 T cells that are $\mathrm{CD} 25^{+} \mathrm{CD} 127^{\mathrm{lo}} \mathrm{FoxP}^{+}(\mathrm{n}=3)$, and the representative dot plots show the frequency of cell expressing $\mathrm{CD} 25$ and FoxP3 within the $\mathrm{CD} 4^{+} \mathrm{CD} 127^{\mathrm{lo}}$ population. (B) Tritiated thymidine was added for the final $18 \mathrm{~h}$ to measure proliferation, and the concentrations of IFN- $\gamma$ present in the supernatants were determined by ELISA. (C) Proliferation of the CFSE-labeled vehicle control-treated cells was assessed by flow cytometry. Representative histograms show proliferation of cells which underwent primary allogeneic stimulation in the presence of vehicle control $\left(\mathrm{CFSE}^{\mathrm{lo}}\right)$ in response to secondary allogeneic stimulation in cells cultured alone and in coculture with cells previously treated with PCB. Statistical significance was assessed by a Student paired $t$ test; however, the results were not statistically significant. PBMC, peripheral blood mononuclear cell.

that BV and PCB may have direct effects on CD4 $\mathrm{T}$ cells. However, because the alloresponse system requires allogeneic stimulation with irradiated total PBMC, effects via antigen presenting cells cannot definitively be ruled out. Therefore, CD4 T cells were sorted and pretreated with $\mathrm{BV}$ and $\mathrm{PCB}$ before stimulating with anti-CD3 and anti-CD28. Proliferation was assessed by incorporation of tritiated thymidine (Fig 3, E). Interestingly, both BV and PCB directly reduced the proliferation of CD4 $\mathrm{T}$ cells; however, BV exhibited significantly enhanced ability to directly downmodulate CD4 $\mathrm{T}$ cell proliferation compared with PCB. The concentrations of IFN- $\gamma$ and IL-17 present in supernatants were determined by
ELISA (Fig 3,F). Both BV and PCB significantly reduced IFN- $\gamma$ production directly from CD4 T cells and a trend toward reduced IL-17 production was observed. These data demonstrate that BV and PCB can exert their immunosuppressive effects directly on CD4 T cells.

Phycocyanobilin does not significantly promote Treg cells. Having demonstrated that $\mathrm{BV}$ and $\mathrm{PCB}$ can directly modulate CD4 T cells resulting in significantly attenuated inflammatory responses to allogeneic stimuli, we next sought to determine if $\mathrm{T}$ cells exposed to these linear tetrapyrroles acquire suppressive capacity thereby resulting in the observed reduction in proinflammatory responses. To assess this, a suppression 

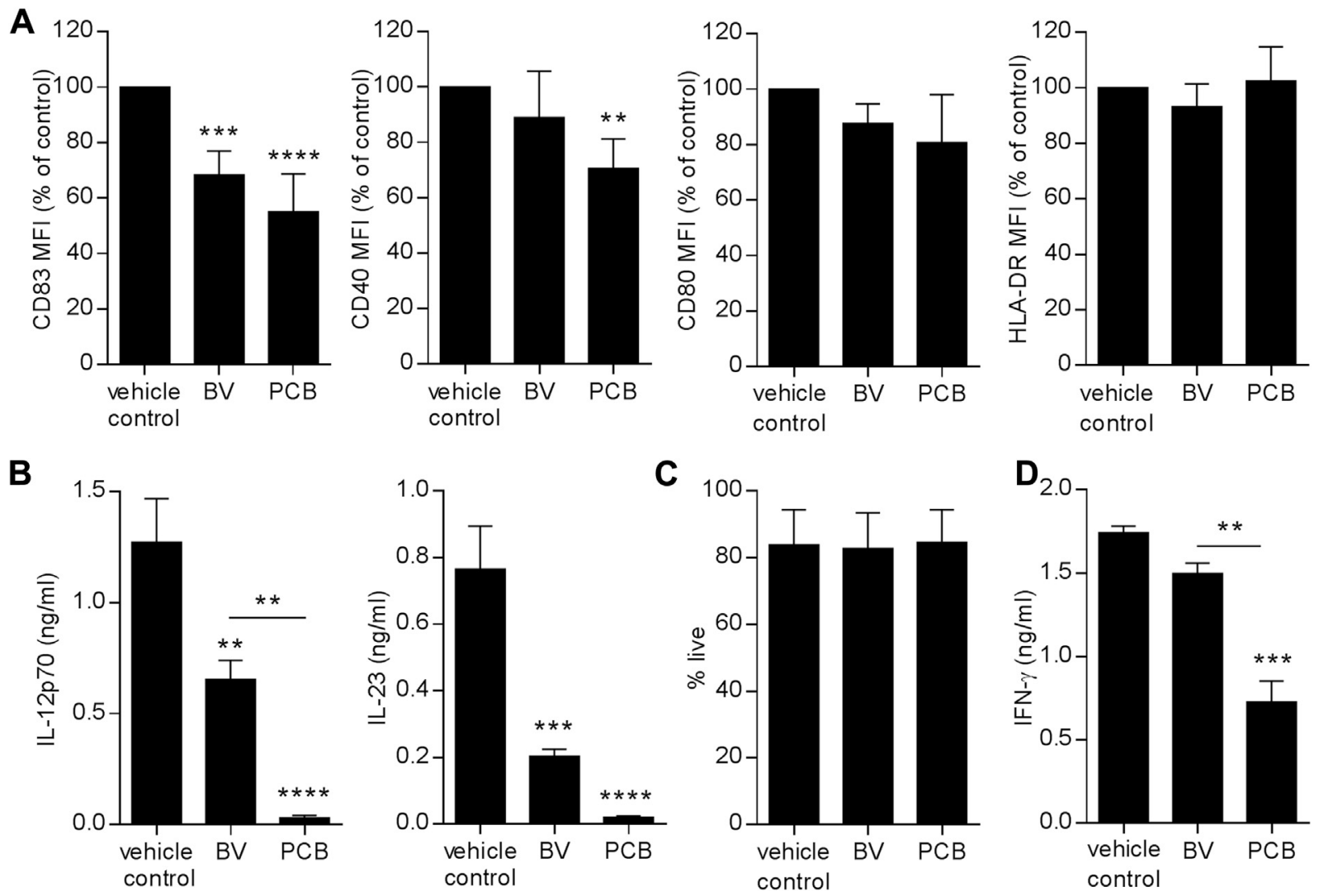

Fig 5. Biliverdin and phycocyanobilin exert anti-inflammatory effects directly on human DC. CD $14^{+}$monocytes were purified from the PBMC of healthy donors $(n=5)$ and differentiated into DC with GM-CSF and IL-4. On day 6 , cells were treated with BV, PCB, or vehicle control for $6 \mathrm{~h}$ before the addition of LPS for $24 \mathrm{~h}$. Cells were stained with fluorochrome-conjugated antibodies specific for CD83, CD40, CD80, and HLA-DR and analyzed by flow cytometry (A). The concentrations of IL-12p70 and IL-23 in the supernatants were determined by ELISA (B). DC viability was assessed after 24-h stimulation with LPS in the presence of BV or PCB (C). DC pretreated with BV or PCB and stimulated with LPS for $24 \mathrm{~h}$ were washed and cocultured with allogeneic CD4 T cells for $5 \mathrm{~d}$. The concentrations of IFN- $\gamma$ present in the supernatants were determined by ELISA (D). Statistical significance was determined by 1 -way ANOVA with Tukey's multiple comparisons post-test; $* * * * P<.0001$, $* * * P<.001, * * P<.01$. PBMC, peripheral blood mononuclear cell; DCs, dendritic cells.

assay was set up as previously described. ${ }^{11}$ Human PBMC were pretreated with PCB or vehicle control before stimulation with irradiated allogeneic PBMC as aforementioned to initiate a primary alloresponse. After 12 days, cells were washed and vehicle controltreated cells were labeled with CFSE and PCB-treated cells were left unlabeled. CFSE-labeled cells were cultured alone or in coculture with cells that had undergone the primary alloresponse in the presence of PCB. Cells were restimulated with the same irradiated allogeneic PBMC used in the primary alloresponse. The proliferation and cytokine production of vehicle control-treated cells cultured alone were compared with vehicle control-treated cells cocultured with cells previously treated with phycocyanbilin to determine if PCB-treated cells displayed suppressive capacity. In addition, the frequencies of Treg cells $\left(\mathrm{CD} 4^{+}\right.$
$\mathrm{CD} 127^{\text {lo }} \mathrm{CD}^{2} 5^{+} \mathrm{FoxP}^{+}$) were assessed in both populations. Cells treated with PCB during the primary alloresponse exhibited a trend toward increased expression of Treg cell markers compared with vehicle control-treated cells; however, this result was not statistically significant (Fig 4, A). Proliferation was assessed by incorporation of tritiated thymidine (Fig 4, B). Vehicle control-treated cells cultured alone proliferated more and produced more IFN- $\gamma$ than when in coculture with cells that had previously been treated with PCB; however, this was not statistically significant. To assess the proliferation of vehicle control-treated cells in the coculture system independently of PCB treated cells, CFSE-labeled cells were gated and dilution of the CFSE label was assessed by flow cytometry. Representative histograms show that $46.4 \%$ of the cells treated with vehicle 
control during the primary alloresponse proliferate in response to secondary allorestimulation, whereas only $30.3 \%$ of these cells proliferate when in coculture with cells that were treated with PCB during the primary alloresponse, indicating that the presence of PCB-treated cells suppressed the secondary restimulation of vehicle control-treated cells by $34.7 \%$ (Fig 4, C). These results indicate that cells treated with PCB during the primary alloresponse may acquire an increased tendency toward suppressive capacity but do not exhibit a significant increase in the frequencies of Treg cells present.

Biliverdin and $\mathrm{PCB}$ directly modulate dendritic cell responses resulting in reduced allospecific Th1 responses. DCs play a key role in bridging the innate and adaptive response by presenting antigens in the context of MHC-II to the TCR of naive CD4 T cells. Furthermore, DCs provide costimulation and instructive cytokines to polarize the naive CD4 T cell into an appropriate effector cell. Organ rejection following transplantation is mediated by recipient alloreactive $\mathrm{T}$ cells which can be activated directly by donor DC from the transplanted tissue or indirectly by recipient DC. In both scenarios, the DC is instrumental in shifting the immune response toward either tolerance or acute rejection. To determine if $\mathrm{BV}$ and PCB reduce alloinduced inflammation by tolerising the innate antigen presenting $\mathrm{DC}$, human monocyte-derived DC were treated with $50-\mu \mathrm{M} \mathrm{BV}$, PCB, or vehicle control before stimulating with LPS for 24 hours. The expression of costimulatory molecules CD83, CD80, and CD40 were analyzed by flow cytometry, as was the expression of HLA-DR, to determine if antigen presentation is effected by the compounds. The concentrations of instructive cytokines IL-12p70 and IL-23 were measured in cell supernatants. BV and $\mathrm{PCB}$ significantly reduced the expression of CD83 on DC, whereas only PCB significantly reduced CD40 expression (Fig 5, A). No significant effect was seen on CD80 or HLA-DR. The concentrations of IL-12p70 and IL-23 were significantly reduced in DC treated with either BV or PCB (Fig 5, B) with PCB having a more potent effect on IL-12p70 when compared with BV. These data indicate that, in addition to directly modulating CD4 $\mathrm{T}$ cell responses, $\mathrm{BV}$ and $\mathrm{PCB}$ can directly downregulate the inflammatory response in innate DC. Moreover, these data indicate that PCB exerts more potent effects on DC compared with BV.

Since BV and PCB downregulated the maturation of DC (as marked by their reduced expression of costimulatory molecules) and inhibited their production of polarizing cytokines; IL-12 and IL-23, next we sought to determine if the DC phenotype elicited by the pres- ence of PCB or BV during stimulation would then have a downstream effect on allogeneic CD4 T cell responses in the absence of BV and PCB. IL-12p70 produced by $\mathrm{DC}$ is known to drive adaptive Th1 cell responses which include the production of IFN- $\gamma$. DCs were, therefore, treated with 50- $\mu \mathrm{MBV}, \mathrm{PCB}$, or vehicle control before stimulating with LPS for 24 hours, as above. Cells were then washed and cocultured with allogeneic CD4 T cells at a ratio of $1 \mathrm{DC}$ to $10 \mathrm{~T}$ cells. On day 5 , the concentrations of IFN- $\gamma$ in the supernatants were measured by ELISA (Fig 5, $C$, right). DC treated with PCB exhibited significantly attenuated IFN- $\gamma$ production from CD4 T cells. BV-treated DC exhibited a trend toward reduced IFN- $\gamma$ production by T cells; however, PCB, was significantly more potent at reducing Th1 cell polarization via the DC.

\section{DISCUSSION}

A number of studies in mice have highlighted the protective role of $\mathrm{BV}$ and bilirubin in IRI and organ transplantation. There have, however, been no studies to date examining the effects of linear tetrapyrroles on human alloresponses. In this study, we provide novel data illustrating that mammalian BV and algae-derived $\mathrm{PCB}$ have potent anti-inflammatory effects on human cells. Moreover, these data suggest that in the context of human organ transplantation, these compounds may have improved clinical efficacy for the preoperative and postoperative treatment of patients receiving allografts as they can combat harmful reactive oxygen species production during IRI as well as dampen deleterious proinflammatory responses that lead to acute/chronic rejection.

The anti-inflammatory and cytoprotective properties of the heme-oxygenase system have been observed in a number of disease models including collageninduced arthritis, psoriasis, and experimental autoimmune encephalomyelitis, a murine model of multiple sclerosis. $^{12-14}$ In allogeneic heart transplant experiments, overexpression of HO-1, either by adenoviral transfer or chemical induction by cobalt protoporphyrin IX, increases long-term survival. The exact mechanism by which this occurs remains unknown but it does rely on the enzymatic ability to produce $\mathrm{CO}$ and $\mathrm{BV}$ as inhibition of HO-1 by tinprotoporphyrin IX reverses the protective effects. ${ }^{15,16}$ Proceeding from these initial studies, $\mathrm{CO}$ and BV treatment, alone or in combination, was shown to positively benefit the outcome of ischemia/reperfusion injury, ${ }^{17,18}$ inflammation, ${ }^{19,20}$ and allogeneic immune responses during organ transplantion., ${ }^{7,22}$

In a striking report, Yamashita et al. showed that before an allogeneic heart transplantation, short-term 
treatment (3-weeks) of recipient mice with BV induced tolerance to the donor heart. ${ }^{7}$ Significantly, the surgical introduction of a third syngeneic heart at 120 days, in the absence of any further treatment, resulted in full acceptance, clearly indicating that BV promoted long-term tolerance. In our study, we corroborate these findings in human cells in vitro and furthermore demonstrate that marine-derived PCB also elicited similar tolerance to a secondary allogeneic insult. Moreover, we have shown that the induced tolerance to secondary stimulation is specific to the alloantigen as the cells' ability to respond to mitogen is retained. These effects appear to be independent of HO-1 induction but may involve the conversion of $\mathrm{BV}$ and $\mathrm{PCB}$ to their corresponding rubinoid structures given that both compounds are substrates for human BVR-A.

In addition to reducing primary and secondary allospecific proinflammatory responses in total $\mathrm{PBMC}$, we have shown that BV and PCB can exert their effects directly on T cells. In an effort to elucidate the mechanism by which these linear tetrapyrroles are mediating their effects, we investigated if phycocycanobilin reduced proinflammatory mediators by inducing a population of suppressive $\mathrm{T}$ cells as other studies have shown increased Treg cells in alloanergized cells on secondary allorestimualtion. ${ }^{23}$ Whilst a trend toward increased suppressive capacity and increased frequencies of Treg cells was observed in cells treated with PCB compared with vehicle control, this result was not statistically significant indicating that induction of suppressive cells may be a minor mechanism by which PCB reduces primary and secondary alloresponses.

Efforts to define the immune tolerogenic mechanisms of HO- 1 have recently centered on the DC. HO- 1 can inhibit DC maturation caused by various proinflammatory stimuli such as TNF- $\alpha$ and LPS. ${ }^{24}$ We have also found that $\mathrm{BV}$ and PCB can inhibit DC maturation via downregulation of costimulatory markers and inhibit the production of polarizing cytokines; IL-12 and IL23. Moreover, our data demonstrate that the effect of linear tetrapyrroles on the DC plays a key role in modulating downstream adaptive $\mathrm{CD} 4 \mathrm{~T}$ cell responses. Interestingly, we found that BV and PCB had no effect on the expression of HLA-DR. These data suggest that although antigen presentation is not directly inhibited via the downregulation of HLA-DR expression, T cell activation is likely reduced due to decreased costimulatory molecules and decreased production of polarizing cytokines in DC treated with BV or PCB. Treating DC with PCB before coculturing with allogeneic CD4 $\mathrm{T}$ cells prevented the induction of harmful allospecific Th1 responses which mediate acute and chronic transplant rejection. Therefore, these data suggest that PCB may have therapeutic efficacy in the clinical setting of organ transplantation.

Despite being a major breakthrough in modern medicine, successful organ transplantation simply cannot be carried out in the absence of immunosuppressants. Unfortunately all of these agents exhibit substantial toxicities and drug interactions. There has been a gap in the market over the last decade in the generation of new therapies with safer side-effect profiles and most, if not all, are associated with increased risk of cancer and various types of microbial infection. Prevention and treatment of acute organ rejection therefore remains a major clinical focus and given the limited number of donor organs available, prolonging the lifetime of a graft by preventing damage during the transplantation procedure itself or by preventing acute/chronic rejection is of paramount importance. The identification of naturally derived anti-inflammatory products with minimal side effects, yet which are effective in blocking pathways leading to chronic inflammation is sought-after. Many studies report that the consumption of bluegreen algae promotes immunity and protects against a range of inflammatory diseases such as colitis, arthritis, and allergic rhinitis in animals and humans., ${ }^{9,25-31}$ Encouragingly, we have assessed the tolerability of PCB in vivo and no obvious adverse effects were noted. Assuming that bioavailability is not a factor, algae-derived PCB (or derivatives of PCB) may represent a viable candidate for use in preventative medicines or as therapeutic which could potentially complement existing immunosuppressive treatments. Furthermore, given that $\mathrm{BV}$ and $\mathrm{PCB}$ effectively downregulate maturation and cytokine production from innate antigen presenting DC as well as strongly suppressing proinflammatory responses to $\mathrm{T}$ cell stimulation; it is possible that these compounds may be efficacious, not only in organ transplantation but also in a number of autoinflammatory and autoimmune conditions.

\section{ACKNOWLEDGMENTS}

Conflicts of Interest: All authors have read the journal's policy on disclosure of potential conflicts of interest and have none to declare.

All authors have read the journal's authorship agreement and that the manuscript has been reviewed and approved by all named authors. This work was funded by Science Foundation Ireland (grant no: 14/TIDA/ 2267).

\section{REFERENCES}

1. Nuhn P, Mitkus T, Ceyhan GO, et al. Heme oxygenase 1generated carbon monoxide and biliverdin attenuate the course of experimental necrotizing pancreatitis. Pancreas 2013;42: $265-71$. 
2. Ito T, Chen D, Chang CW, et al. Mesobiliverdin IXalpha enhances rat pancreatic islet yield and function. Front Pharmacol 2013; 4:50.

3. Kosaka J, Morimatsu H, Takahashi T, et al. Effects of biliverdin administration on acute lung injury induced by hemorrhagic shock and resuscitation in rats. PLoS One 2013;8: e63606.

4. Sugimoto R, Tanaka Y, Noda K, et al. Preservation solution supplemented with biliverdin prevents lung cold ischaemia/reperfusion injury. Eur J Cardiothorac Surg 2012;42:1035-41.

5. Wang J, Zhou HC, Pan P, Zhang N, Li WZ. Exogenous biliverdin improves the function of lung grafts from brain dead donors in rats. Transplant Proc 2010;42:1602-9.

6. Tang LM, Wang YP, Wang K, et al. Exogenous biliverdin ameliorates ischemia-reperfusion injury in small-for-size rat liver grafts. Transplant Proc 2007;39:1338-44.

7. Yamashita K, McDaid J, Ollinger R, et al. Biliverdin, a natural product of heme catabolism, induces tolerance to cardiac allografts. FASEB J 2004;18:765-7.

8. Kato Y, Shimazu M, Kondo M, et al. Bilirubin rinse: a simple protectant against the rat liver graft injury mimicking heme oxygenase-1 preconditioning. Hepatology 2003;38:364-73.

9. Romay C, Armesto J, Remirez D, Gonzalez R, Ledon N, Garcia I. Antioxidant and anti-inflammatory properties of C-phycocyanin from blue-green algae. Inflamm Res 1998;47:36-41.

10. Terry MJ, Maines MD, Lagarias JC. Inactivation of phytochromeand phycobiliprotein-chromophore precursors by rat liver biliverdin reductase. J Biol Chem 1993;268:26099-106.

11. Basdeo SA, Moran B, Cluxton D, et al. Polyfunctional, pathogenic CD161+ Th17 lineage cells are resistant to regulatory $t$ cell-mediated suppression in the context of autoimmunity. J Immunol 2015;195:528-40.

12. Ma LJ, You Y, Bai BX, Li YZ. Therapeutic effects of heme oxygenase-1 on psoriasiform skin lesions in guinea pigs. Arch Dermatol Res 2009;301:459-66.

13. Benallaoua M, Francois M, Batteux F, et al. Pharmacologic induction of heme oxygenase 1 reduces acute inflammatory arthritis in mice. Arthritis Rheum 2007;56:2585-94.

14. Liu Y, Zhu B, Wang X, et al. Bilirubin as a potent antioxidant suppresses experimental autoimmune encephalomyelitis: implications for the role of oxidative stress in the development of multiple sclerosis. J Neuroimmunol 2003; 139:27-35.

15. Araujo JA, Meng L, Tward AD, et al. Systemic rather than local heme oxygenase-1 overexpression improves cardiac allograft outcomes in a new transgenic mouse. J Immunol 2003;171: 1572-80.

16. Uchida Y, Tamaki T, Tanaka M, et al. Induction of specific stress response increases resistance of rat liver allografts to cold ischemia and reperfusion injury. Transpl Int 2003;16: 396-404.
17. Zhang X, Shan P, Otterbein LE, et al. Carbon monoxide inhibition of apoptosis during ischemia-reperfusion lung injury is dependent on the p38 mitogen-activated protein kinase pathway and involves caspase 3. J Biol Chem 2003;278:1248-58.

18. Adin CA, Croker BP, Agarwal A. Protective effects of exogenous bilirubin on ischemia-reperfusion injury in the isolated, perfused rat kidney. Am J Physiol Renal Physiol 2005;288: F778-84.

19. Ott MC, Scott JR, Bihari A, et al. Inhalation of carbon monoxide prevents liver injury and inflammation following hind limb ischemia/reperfusion. FASEB J 2005;19:106-8.

20. Berberat PO, AR YI, Yamashita K, et al. Heme oxygenase-1generated biliverdin ameliorates experimental murine colitis. Inflamm Bowel Dis 2005;11:350-9.

21. Nakao A, Otterbein LE, Overhaus M, et al. Biliverdin protects the functional integrity of a transplanted syngeneic small bowel. Gastroenterology 2004;127:595-606.

22. Fondevila C, Shen XD, Tsuchiyashi S, et al. Biliverdin therapy protects rat livers from ischemia and reperfusion injury. Hepatology 2004;40:1333-41.

23. Davies JK, Barbon CM, Voskertchian A, Nadler LM, Guinan EC. Ex vivo alloanergization with belatacept: a strategy to selectively modulate alloresponses after transplantation. Cell Transplant 2012;21:2047-61.

24. Chauveau C, Remy S, Royer PJ, et al. Heme oxygenase- 1 expression inhibits dendritic cell maturation and proinflammatory function but conserves IL-10 expression. Blood 2005;106:1694-702.

25. Selmi C, Leung PS, Fischer L, et al. The effects of spirulina on anemia and immune function in senior citizens. Cell Mol Immunol 2011;8:248-54.

26. Mao TK, Van de Water J, Gershwin ME. Effects of a spirulinabased dietary supplement on cytokine production from allergic rhinitis patients. J Med Food 2005;8:27-30.

27. Rasool M, Sabina EP, Lavanya B. Anti-inflammatory effect of spirulina fusiformis on adjuvant-induced arthritis in mice. Biol Pharm Bull 2006;29:2483-7.

28. Park YK, Rasmussen HE, Ehlers SJ, et al. Repression of proinflammatory gene expression by lipid extract of Nostoc commune var sphaeroides Kutzing, a blue-green alga, via inhibition of nuclear factor-kappaB in RAW 264.7 macrophages. Nutr Res 2008;28:83-91.

29. Ku CS, Pham TX, Park Y, et al. Edible blue-green algae reduce the production of pro-inflammatory cytokines by inhibiting NFkappaB pathway in macrophages and splenocytes. Biochim Biophys Acta 2013;1830:2981-8.

30. Deng R, Chow TJ. Hypolipidemic, antioxidant, and antiinflammatory activities of microalgae spirulina. Cardiovasc Ther 2010;28: e33-45.

31. Gonzalez R, Rodriguez S, Romay C, et al. Anti-inflammatory activity of phycocyanin extract in acetic acid-induced colitis in rats. Pharmacol Res 1999;39:1055-9. 


\section{Appendix}
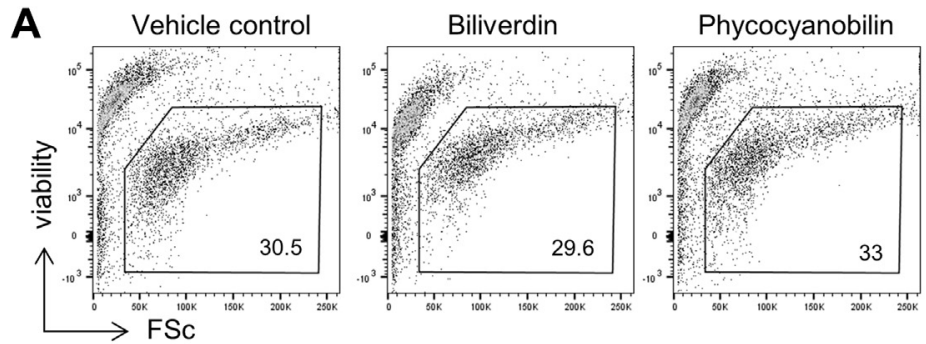

B

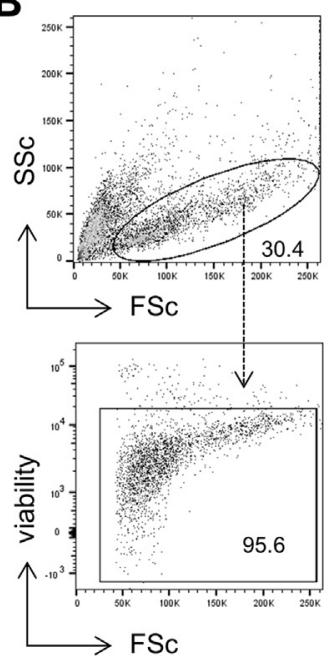

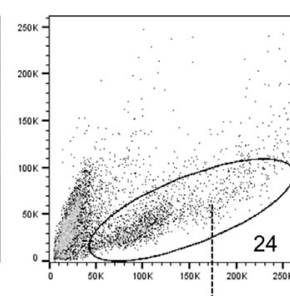
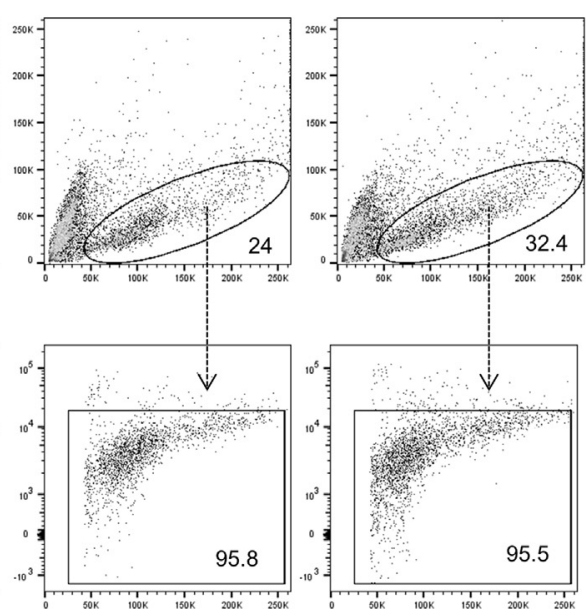

C

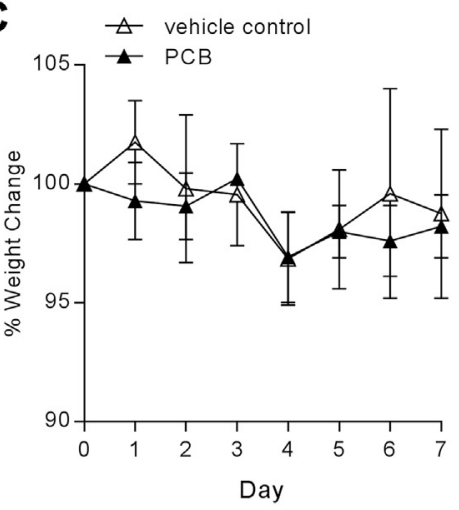

D

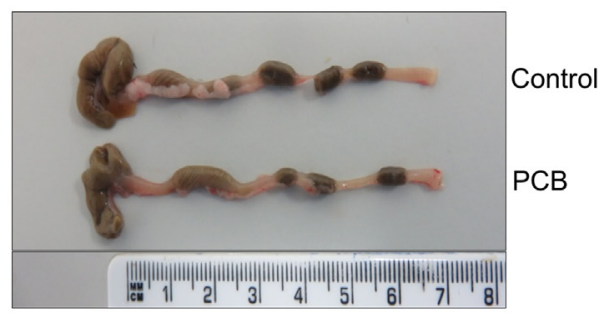

Supplemental Fig 1. Biliverdikn and phycocyanobilin are nontoxic in vitro and in vivo. Human PBMC were isolated and treated with biliverdin (BV), phycocyanobilin (PCB), or vehicle control (Tris $\mathrm{HCl}$ at pH 7.4) for $6 \mathrm{~h}$ before stimulation with anti-CD3 for $72 \mathrm{~h}$. Cells were stained with a viability dye and analyzed by flow cytometry. (A) Ungated cells showing the staining of dead cells (viability dye ${ }^{\text {high }}$ ) vs forward scatter (FSc). (B) For viability analysis, lymphocytes were gated on the basis of FSc and side scatter (SSc) and viability dye ${ }^{\text {lo }}$ cells were gated to give the frequency of live lymphocytes in each treatment group (B; lower panel). (C) C57B1/6 mice were treated with either PCB $(30 \mathrm{mg} / \mathrm{kg}$ ) or control (PBS) on days 0,3 , and 5 by oral gavage. Mice were sacrificed on day 7 . (C) Percentage weight change during 7-day trial. (D) Representative macroscopic view of dissected colons. PBMC, peripheral blood mononuclear cell 

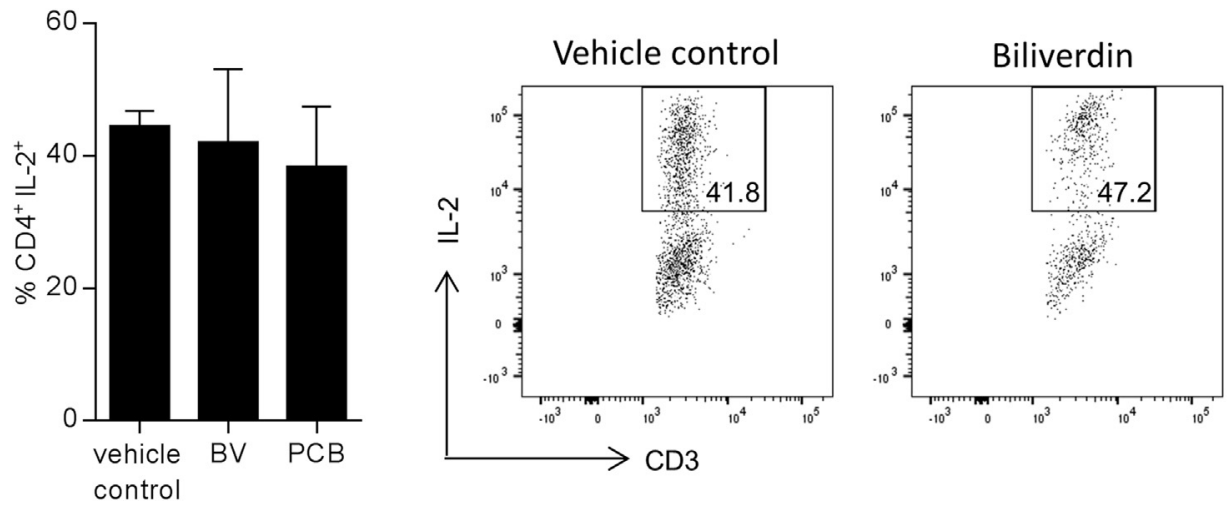

\section{Phycocyanobilin}

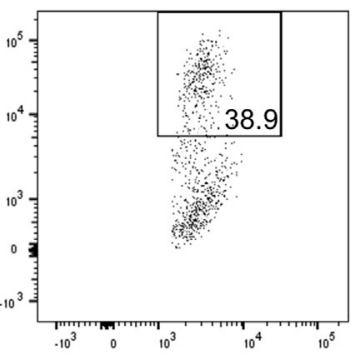

Supplemental Fig 2. Biliverdi)phorbol 12-myristate 13-acetate (n and phycocyanobilin do not affect the ability of $\mathrm{T}$ cells to produce IL-2. PBMC were isolated from healthy donors $(\mathrm{n}=4)$ and treated with biliverdin $(\mathrm{BV})$, phycocyanobilin (PCB), or vehicle control (Tris $\mathrm{HCl}$ at $\mathrm{pH}$ 7.4) for $6 \mathrm{~h}$ before stimulation with anti-CD3 for 72 hours. Cells were restimulated with phorbol 12-myristate 13-acetate (PMA) and ionomycin in the presence of brefeldin A for 5 hours and stained with fluorochrome-conjugated antibodies specific for CD3, CD8, and IL2. Cells were analyzed by flow cytometry; lymphocytes were gated on the basis of forward and side scatter and $\mathrm{CD} 4 \mathrm{~T}$ cells were identified as $\mathrm{CD} 3^{+} \mathrm{CD} 8^{-}$. The graph show the collated data $(\mathrm{n}=4)$ of the frequencies of CD4 T cells producing IL-2. Representative dot plots show the staining of IL-2. Statistical significance was determined by 1-way ANOVA; however, these results were not significantly different. PBMC, peripheral blood mononuclear cell. 